\title{
Efeito da somatotropina recombinante bovina (rbST) sobre os níveis de marcadores metabólicos de touros semiconfinados das raças Aberdeen Angus e Brangus
}

\author{
Effect of recombinant bovine somatotropin (rbST) on the \\ concentrations of metabolic markers in semi-confined sires \\ Aberdeen Angus and Brangus
}

\author{
Marcelo Brandi Vieira, ${ }^{*}$ Vinícius Coitinho Tabeleão, ${ }^{*}$ Elizabeth Schwegler, ${ }^{*}$ Maikel Alan Goulart, ${ }^{*}$ \\ Viviane Rohrig Rabassa, ${ }^{*}$ Augusto Schneider, ${ }^{*}$ Francisco Augusto Burket del Pino, ${ }^{* *}$ Víctor Fernando Buttow Roll, ${ }^{* * *}$ \\ Carlos Eduardo Wayne Nogueira, ${ }^{*}$ Marcio Nunes Corrêa*
}

\begin{abstract}
Resumo
O objetivo deste estudo foi avaliar o efeito da somatotropina recombinante bovina (rbST) sobre os marcadores do metabolismo energético, proteico, mineral e hepático de touros mantidos em semiconfinamento. Foram utilizados 20 touros de raças Aberdeen Angus e Brangus, sendo divididos aleatoriamente em dois grupos (Placebo e rbST), para que no Placebo fosse administrado via subcutânea duas doses de $\mathrm{NaCl}, 0,9 \%$ e no rbST fosse administrado o rbST (Lactotropin ${ }^{\circledR}$ ). Foram realizadas cinco coletas de sangue, com intervalo de 14 dias, para avaliação da concentração de triacilgliceróis e colesterol como marcadores energéticos, ureia, como indicador proteico, fosfatase alcalina e fósforo como marcadores minerais e as enzimas aspartato aminotransferase (AST) e a gama glutamiltransferase (GGT) como indicadoras da função hepática. A aplicação de rbST não influenciou os marcadores energéticos, minerais, e proteico, exceto a enzima hepática $G G T(P<0,05)$. Estes resultados indicam que a utilização do rbST em touros não alterou o metabolismo, pois os parâmetros avaliados mantiveram-se dentro dos padrões considerados fisiológicos para a espécie.
\end{abstract}

Palavras-chave: metabolismo, touros, somatotropina recombinante bovina.

\begin{abstract}
The aim of this study was to evaluate the effect of recombinant bovine somatotropin (rbST) on metabolic markers for energy, protein, mineral and hepatic metabolism of sires kept grazing and receiving concentrate. Twenty sires Aberdeen Angus and Brangus used for this experiment were divided in two groups (Placebo and rbST). The group Placebo received subcutaneous administration of $\mathrm{NaCl}, 0.9 \%$ and rbST receive rbST twice. Five blood collections with 14 days of interval were made for evaluation of triglyceride and cholesterol as energetic metabolism markers, urea, as proteic marker, alkaline phosphatase and phosphorus as minerals markers and aspartate aminotransferase (AST) and gamma glutamyltransferase (GGT) as indicators of the hepatic function. The rbST did not influence the energetic, mineral and proteic markers, except the hepatic enzyme GGT $(P<0,05)$. These results indicated that the use of rbST in sires did not affect metabolism, therefore evaluated parameters remaining in the physiological range for the specie.
\end{abstract}

Keywords: Metabolism, sires, recombinant bovine somatotropin.

\section{Introdução}

Estudos relacionados com o efeito da somatotropina (hormônio do crescimento - GH) começaram a ser desenvolvidos próximo ao ano de 1980, com a síntese em larga escala da forma recombinante deste hormônio (rbST), sendo utilizado para tratamento de desordens do crescimento em humanos ou para a estimulação da produção de leite e carne em animais de produção (Sirotkin, 2005).

O GH está associado com o crescimento e desenvolvimento celular, bem como com processos metabólicos, sendo descrito como um agente anabólico (Prado et al., 2003), desempenhando um importante papel no controle do metabolismo energético e proteico (Kaiser et al., 2001). Sua ação é mediada pelo fator de crescimento semelhante à insulina I (IGF-I), que é secretado por inúmeras células mitogênicas, sendo que o fígado é o órgão responsável pela sua maior produção (Breier, 1999). Estudos conduzidos com bovinos demonstram que o tratamento com rbST aumentou a massa muscular e diminuiu a deposição de gordura (Kolb, 1984; Breier, 1999). O GH aumenta ainda a retenção de nitrogênio e fósforo para a produção de adenosina tri-fosfato (ATP) (Kolb, 1984).

\footnotetext{
* Universidade Federal de Pelotas (UFPel), Faculdade de Veterinária, Departamento de Clínicas Veterinária-NUPEEC, CEP: 96010-900, Pelotas, RS, Brasil.

** Departamento de Bioquímica, UFPel.

*** Faculdade de Agronomia Eliseu Maciel, UFPel.

A quem enviar correspondência: Marcelo Brandi Vieira / Marcio Nunes Corrêa - m-vieira@ufpel.edu.br, www.ufpel.edu.br/nupeec
} 
Outra importante função do GH é sua participação nos processos reprodutivos, mediado por receptores nas gônadas masculinas e femininas (Hull e Harvey, 2000; Gong, 2002; Marchal et al., 2003; Sirotkin, 2005). Estudos têm-se concentrado nas interações entre o metabolismo e o desempenho reprodutivo de bovinos, principalmente em fêmeas (Butler, 2000; Gong, 2002), e com menor intensidade em touros. Além disso, em animais de produção, com adequada fertilidade, os efeitos da suplementação de $\mathrm{GH}$ não estão claros, havendo alguns estudos realizados em garanhões (Storer et al., 2005) e touros (Sauerwein et al., 2000), que demonstram efeito sobre o perfil hormonal, através do aumento nos níveis séricos de GH, IGF-I e insulina, e aumento da qualidade espermática, caracterizado por um incremento na taxa de fertilização. Porém, as consequências desta suplementação sobre o metabolismo destes animais não estão bem esclarecidas.

As concentrações dos metabólitos sanguíneos representam um indicador imediato da condição metabólica (PambuGollah et al., 2000), sendo uma importante ferramenta para detectar alguns distúrbios metabólicos capazes de gerar prejuízos à saúde animal, fertilidade e a capacidade produtiva do rebanho (Payne e Payne, 1987).

Assim, este estudo visa determinar se a administração de rbST altera ou não o metabolismo de touros, de forma a permitir o seu uso visando melhorar parâmetros ligados à qualidade espermática de touros. O objetivo do presente estudo foi avaliar os níveis de alguns marcadores energéticos (colesterol e triglicerídeos - TAG), proteico (ureia), minerais (fósforo e fosfatase alcalina) e hepáticos (aspartato aminotransferase - AST e gama-glutamiltransferase - GGT) de touros tratados com rbST mantidos em sistema de semiconfinamento.

\section{Material e métodos}

Este estudo foi realizado em uma central de inseminação artificial situada no município de Rosário do Sul, RS, Brasil, localizado a 3015'28' Sul e 5454'50" Oeste, durante os meses de fevereiro a abril, com temperatura ambiente média de 23,7ํㅡ (máxima: 31,0ํㅜ ; mínima: 15,6ํㅡ). Foram utilizados touros adultos, sendo todos mantidos nas mesmas condições ambientais e nutricionais e com o mesmo regime de coleta de sêmen. A dieta fornecida aos animais era calculada de acordo com as normas do National Research Council (1998) para esta determinada categoria animal, sendo constituída de concentrado $(88,41 \%$ de matéria seca, $18,63 \%$ de proteína bruta, $8,02 \%$ de cinzas, $2,91 \%$ de extrato etéreo, $7,22 \%$ de fibra bruta, 45,52\% de FDN, 8,34\% de FDA, 1,34\% de cálcio e $0,83 \%$ de fósforo) e feno de aveia e azevém ( $89,30 \%$ de matéria seca, $13,00 \%$ de proteína bruta, $10,18 \%$ de cinzas, $2,01 \%$ de extrato etéreo, 32,34\% de fibra bruta, 61,19\% de FDN, 35,31\% de FDA, 0,13\% de cálcio e $0,032 \%$ de fósforo).

Foram selecionados 20 touros (10 touros Aberdeen Angus e 10 touros Brangus), com condição corporal média de 4,0 (escala de 1,0 a 5,0), divididos em dois grupos de 10 animais, sendo 5 animais da raça Aberdeen Angus e 5 Brangus. $O$ grupo Placebo recebeu por via subcutânea duas doses de placebo ( $\mathrm{NaCl}$ 0,9\%) e o grupo rbST recebeu 500 mg/animal de rbST (Lactotropin ${ }^{\circledR} /$ Elanco Saúde Animal). Na mesma frequência e via de administração, com intervalo de 14 dias entre as aplicações do produto, foi determinado considerando a liberação e ação lenta do rbST (Prado et al., 2003).

Foram realizadas 5 coletas de sangue para a avaliação dos marcadores, energéticos (colesterol e TAG), proteico (ureia), minerais (fósforo e fosfatase alcalina) e hepáticos (AST e GGT) (González et al., 2000). Estas coletas iniciaram no dia da primeira aplicação de rbST e continuaram a cada 14 dias até o dia 56 do experimento.

As amostras de sangue foram coletadas de todos os animais no turno da manhã, através de punção da veia jugular, sendo divididas em 2 tubos: tubo 1, contendo anticoagulante (EDTA $10 \mathrm{~g} \%$ ) na proporção de $12 \mu \mathrm{L} / \mathrm{mL}$ de sangue e tubo 2 , sem anticoagulante. Posteriormente, as amostras foram centrifugadas a $3500 \mathrm{~g}$ durante 15 minutos e armazenadas em dois microtubos, dos quais um foi congelado a $-20^{\circ} \mathrm{C}$ e 0 outro resfriado $\mathrm{a}+4^{\circ} \mathrm{C}$. O período decorrido entre a coleta das amostras e o seu processamento foi de no máximo 12 horas. Foi utilizado método colorimétrico para determinação dos níveis sanguíneos de colesterol (Colesterol Liquiform Labtest Diagnóstica S.A., Brasil), TAG (Triglicérides Liquiform - Labtest Diagnóstica S.A., Brasil), ureia (Ureia CE - Labtest Diagnóstica S.A., Brasil), fósforo (Fósforo - Labtest Diagnóstica S.A., Brasil), fosfatase alcalina (Fosfatase Alcalina Liquiform Labtest Diagnóstica S.A., Brasil), AST (AST/GOT Liquiform Labtest Diagnóstica S.A., Brasil) e GGT (Gama GT Liquiform Labtest Diagnóstica S.A., Brasil), utilizando espectrofotômetro de luz visível (FEMTO 435 ${ }^{\circledR}$, Brasil).

As concentrações séricas do colesterol foram determinadas nas amostras congeladas $\left(-20^{\circ} \mathrm{C}\right)$ e as análises de TAG, assim como o fósforo, fosfatase alcalina e ureia foram realizadas nas amostras de soro mantidas em microtubos a $\pm 4^{\circ} \mathrm{C}$. A enzima AST foi determinada em soro refrigerado e a GGT nas amostras congeladas.

Durante todas as coletas, foram realizadas avaliações do escore de condição corporal (ECC) de todos os animais utilizando a escala numérica de 1 a 5 (sendo $1=$ magro e $5=$ gordo), conforme Lowman et al. (1976), sendo descritas suas frequências.

As análises estatísticas foram realizadas utilizando-se 0 procedimento GLM num modelo fatorial de efeitos fixos: $Y_{i j k}=$ $\mu+A_{i}+\beta_{i}+(A \beta)_{i j}+E_{i j k}$ onde: $Y_{i j k}$ é a variável resposta; $\mu$ é a média geral; $A_{i}$ é o efeito do fator tratamento; $\beta$ é o efeito do fator período de coleta; $(A \beta)$ é o efeito da interação (tratamento $x$ coleta); e $E_{i j k}$ é o erro aleatório. Para os parâmetros que apresentaram na análise de variância valores de $\mathrm{P}<0,05$, foi aplicado o teste de Tukey HSD para comparação entre médias.

Para predizer o efeito do período de coleta sobre os parâmetros metabólicos, em cada grupo experimental foi utilizada análise de regressão polinomial, sendo os modelos escolhidos de acordo com a significância dos coeficientes de regressão $(P<0,05)$ e pelo coeficiente de determinação.

\section{Resultados}

Os efeitos do tratamento com rbST sobre as médias dos marcadores bioquímicos não foram relevantes (Tabela 1), sendo encontrada apenas diferença $(P<0,05)$ para a enzima hepática GGT, em que no grupo rbST foram observadas maiores concentrações. 
Houve diferença entre coletas para as concentrações de ureia, colesterol, TAG, AST e GGT, enquanto as concentrações da fosfatase alcalina e fósforo não variaram ao longo do período (Tabela 1).

Também foram encontradas diferenças na interação coleta $x$ tratamento nos níveis de ureia, TAG, AST e GGT. Nas Figuras 1 e 2 são apresentadas as análises de regressão para os durante todo o período do experimento. Ambos os grupos apresentaram maiores concentrações de ureia na coleta do dia 0 (Figura 1), porém, estavam de acordo com os valores fisiológicos (González e Silva, 2003).

O colesterol, que possui importante função metabólica por ser constituinte das membranas celulares, além de ser precursor dos hormônios esteroides (Grummer e Carroll,

Tabela 1: Médias ( \pm EPM) dos marcadores bioquímicos de touros semiconfinados submetidos a tratamento com rbST, de acordo com os tratamentos e períodos de coleta

\begin{tabular}{|c|c|c|c|c|c|c|c|}
\hline \multicolumn{8}{|c|}{ Marcadores Avaliados } \\
\hline & $\begin{array}{c}\text { Ureia } \\
\text { (mg/dL) }\end{array}$ & $\begin{array}{r}\text { Colesterol } \\
(\mathrm{mg} / \mathrm{dL})\end{array}$ & $\begin{array}{c}\text { TAG } \\
(\mathrm{mg} / \mathrm{dL})\end{array}$ & $\begin{array}{c}\text { F.Alcalina } \\
(\mathrm{U} / \mathrm{L})\end{array}$ & $\begin{array}{l}\text { Fósforo } \\
\text { (mg/dL) }\end{array}$ & $\begin{array}{l}\text { AST } \\
(\mathrm{U} / \mathrm{L})\end{array}$ & $\begin{array}{l}\text { GGT } \\
(\mathrm{U} / \mathrm{L})\end{array}$ \\
\hline \multicolumn{8}{|c|}{ Médias de acordo com os grupos } \\
\hline Placebo & $19,2 \pm 0,8^{a}$ & $75,4 \pm 2,0^{\mathrm{a}}$ & $88,8 \pm 3,8^{a}$ & $11,33 \pm 1,19^{a}$ & $0,9 \pm 0,1^{\mathrm{a}}$ & $42,5 \pm 0,5^{\mathrm{a}}$ & $21,2 \pm 1,0^{a}$ \\
\hline rbST & $19,4 \pm 0,8^{\mathrm{a}}$ & $80,2 \pm 2,4^{a}$ & $92,5 \pm 4,3^{\mathrm{a}}$ & $10,19 \pm 0,69^{a}$ & $0,9 \pm 0,2^{\mathrm{a}}$ & $43,4 \pm 0,5^{\mathrm{a}}$ & $27,1 \pm 1,0^{b}$ \\
\hline \multicolumn{8}{|c|}{ Médias de acordo com as coletas } \\
\hline Dia 0 & $25.7 \pm 1.0^{a}$ & $81.1 \pm 3.7^{\mathrm{ab}}$ & $135.1 \pm 4.6^{\mathrm{a}}$ & $10.2 \pm 1.1^{\mathrm{a}}$ & $0.6 \pm 0.02^{a}$ & $39.7 \pm 0.4^{c}$ & $26.9 \pm 1.2^{\mathrm{ab}}$ \\
\hline Dia 14 & $16.9 \pm 0.9^{b}$ & $86.2 \pm 3.8^{\mathrm{a}}$ & $74.8 \pm 3.1^{\mathrm{b}}$ & $8.3 \pm 1.0^{\mathrm{a}}$ & $0.8 \pm 0.03^{\mathrm{a}}$ & $40.9 \pm 0.5^{\mathrm{bc}}$ & $31.7 \pm 1.9^{\mathrm{a}}$ \\
\hline Dia 28 & $19.4 \pm 1.1^{\mathrm{b}}$ & $77.8 \pm 3.2^{\mathrm{ab}}$ & $84.6 \pm 4.8^{b}$ & $8.7 \pm 0.9^{a}$ & $0.9 \pm 0.03^{\mathrm{a}}$ & $45.9 \pm 0.4^{\mathrm{a}}$ & $23.8 \pm 1.6^{\mathrm{bc}}$ \\
\hline Dia 42 & $17.7 \pm 1.3^{b}$ & $69.6 \pm 3.0^{b}$ & $85.4 \pm 1.4^{b}$ & $12.7 \pm 1.9^{a}$ & $1.5 \pm 0.5^{\mathrm{a}}$ & $42.8 \pm 0.8^{b}$ & $19.9 \pm 1.0^{b c}$ \\
\hline Dia 56 & $16.8 \pm 0.8^{b}$ & $74.3 \pm 2.9^{\mathrm{ab}}$ & $73.0 \pm 4.2^{b}$ & $13.9 \pm 2.0^{\mathrm{a}}$ & $0.9 \pm 0.04^{\mathrm{a}}$ & $45.5 \pm 0.5^{a}$ & $18.8 \pm 1.0^{c}$ \\
\hline
\end{tabular}

Médias seguidas por letras diferentes na mesma coluna diferem pelo teste de Tukey $(P<0,05)$.

diferentes períodos de coleta para predição das concentrações destes marcadores em animais tratados ou não com o rbST.

Os níveis de ureia do Placebo, através da equação de regressão, demonstraram efeito quadrático $(P<0,05)$. Já os níveis do grupo rbST demonstraram efeito cúbico $(P<0,05)$. Os níveis de TAG e GGT melhor se ajustaram a um modelo cúbico $(P<0,05)$ ainda que os coeficientes de determinação não sejam muito altos. A enzima AST melhor se ajustou a um modelo linear $(P<0,05)$ em ambos os tratamentos.

Em relação ao ECC, 90\% dos animais apresentaram ECC 4 e $10 \%$ com ECC 5 , as quais foram mantidas durante todo o experimento.

\section{Discussão}

Após análises, a maioria dos marcadores bioquímicos constituintes do perfil metabólico mantiveram-se de acordo com os padrões fisiológicos (Kaneko, 1997; González e Silva, 2003), sendo que apenas os valores de TAG foram superiores e os de fósforo foram considerados abaixo dos padrões normais para a espécie.

As concentrações de ureia, que são indicadoras dos níveis proteicos administrados na dieta e do catabolismo proteico (Wittner et al., 1993), não foram diferentes entre os dois grupos (Tabela 1). O rbST possui ação anabólica, causando diminuição do catabolismo proteico (Breier, 1999). Possivelmente este efeito não tenha sido observado neste estudo, de acordo com os índices de ureia observados, pois os animais estavam em condição metabólica estabilizada, sem degradação de proteínas para ser utilizada como fonte energética e por apresentarem condição corporal elevada,
1991), não apresentou diferenças entre os grupos, demonstrando que o rbST não influenciou este marcador (Tabela 1). O mesmo pode ser observado em estudo conduzido por Prado et al. (2003), com novilhas submetidas à dose única de rbST ou a cada 14 dias. Da mesma forma, Holzer et al. (1999), avaliando dois níveis de energia na dieta e diferentes doses de rbST aplicado a cada 14 dias, não observaram diferença na concentração de colesterol para os tratamentos que receberam doses de rbST. A variação dos níveis de colesterol durante as coletas se manteve dentro do intervalo fisiológico de 80 a $120 \mathrm{mg} / \mathrm{dL}$ (González e Silva, 2003).

Em relação ao TAG, não foram observadas diferenças entre os dois grupos (Tabela 1), porém, os níveis deste marcador se apresentaram elevados, tanto no Placebo quanto no rbST. Provavelmente esta elevação possa ter ocorrido pelo horário das coletas, as quais eram realizadas em torno de três horas após a alimentação destes animais, já que o TAG possui uma grande variação nos seus valores plasmáticos em função do tempo decorrido entre a ingestão de alimento e a coleta de sangue (González e Silva, 2003). Isto se deve à produção de quilomícrons, podendo resultar em aumento temporário nos níveis de TAG (Lehninger et al., 1995); porém, em estudo conduzido com fêmeas, foi observada redução dos níveis de TAG nos animais que receberam o tratamento com rbST (Lapierre et al., 1991), o que não aconteceu neste estudo. Os altos níveis de TAG podem ser explicados pela dieta destes animais, a qual provavelmente foi fornecida em níveis acima do recomendado para a categoria, o que pode ser visto pelo alto ECC dos animais durante o experimento.

A fosfatase alcalina, que é uma enzima indicativa de distúrbios ósseos, apresenta-se elevada em casos de osteoma- 
lácia, hipertireoidismo, tumor ósseo, deficiência de vitamina D e raquitismo (González e Silva, 2003). Neste estudo, os valores deste marcador apresentaram-se estabilizados, não sendo influenciado pela ação do rbST.

O fósforo que possui como principais funções estruturar a matriz óssea e compor as nucleoproteínas e fosfolipídios, não foi influenciado pelo rbST (Tabela 1), mantendo-se estabilizado durante o estudo. Os resultados em ambos os grupos foram abaixo do intervalo fisiológico para bovinos (2,0-9,6 mg/dL - González e Silva, 2003). Em função do GH estimular a síntese proteica, ocorre aumento da retenção de nitrogênio e fósforo para a produção de ATP, podendo gerar diminuição na concentração de fósforo na circulação sanguínea (Kolb, 1984), o que não foi observado neste estudo, pois a diminuição constatada foi nos dois grupos. Animais mantidos em condições de estresse apresentam ainda diminuição significativa nas concentrações de fósforo e outros minerais (Orr, 1990), o que pode ter desencadeado as menores concentrações de fósforo em relação aos níveis fisiológicos observadas neste estudo, já que o experimento foi realizado durante os meses de fevereiro a abril, em que os animais foram submetidos à condições climáticas desfavoráveis, com altas temperaturas. Este efeito também pôde ser observado em um estudo conduzido com fêmeas durante o verão (Vasquez et al., 2003).

A enzima hepática AST, que está associada a problemas hepáticos, sendo também um indicador de lesões musculares, não apresentou diferenças entre os dois grupos, apenas ocorrendo variação durante as coletas (Tabela 1). Já a enzima GGT, com função associada às membranas hepáticas, especialmente as dos epitélios dos ductos biliares (González e Silva, 2003), foi diferente entre os dois grupos, sendo observado maiores valores no rbST $(P<0,05)$ (Tabela 1). Por ser uma enzima caracterizada por alta sensibilidade em resposta a alterações hepáticas (González, 2000), o aumento nas concentrações do rbST pode ser justificado pela ampliação da função hepática, já que o rbST provoca profundas modificações no metabolismo dos lipídeos, aumentando a mobilização, a concentração plasmática (Eiseman et al., 1986) e a taxa de oxidação de ácidos graxos livres (Breier, 1999), utilizados no fígado como via metabólica alternativa (Pámela e Harvey, 1997).

\section{Placebo}

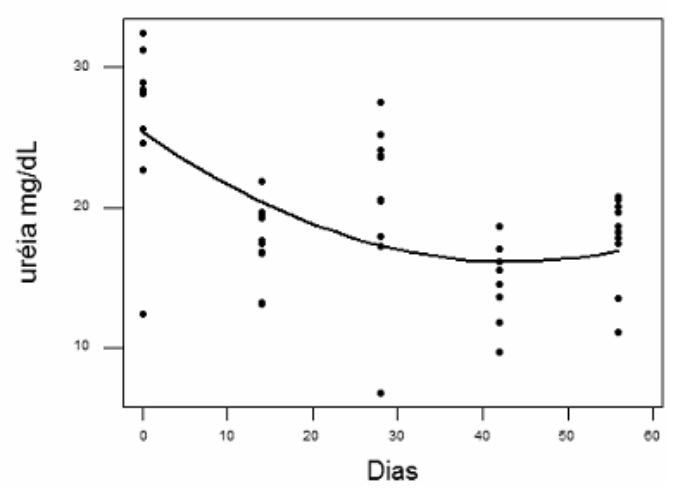

$\mathrm{R}^{2}=34,8 \%$

$\mathrm{Y}=25,7256-0,588391 \mathrm{X}+1,30 \mathrm{E}-02 \mathrm{X} * * 2-9,54 \mathrm{E}-05 \mathrm{X} * * 3$

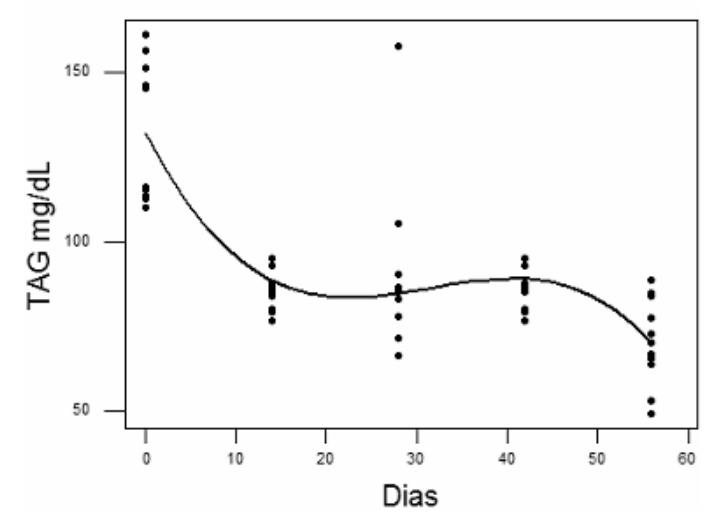

$\mathrm{R}^{2}=63,1 \%$

$\mathrm{Y}=136,226-8,47021 \mathrm{X}+0,306706 \mathrm{X} * 2-3,12 \mathrm{E}-03 \mathrm{X} * * 3$
rbST

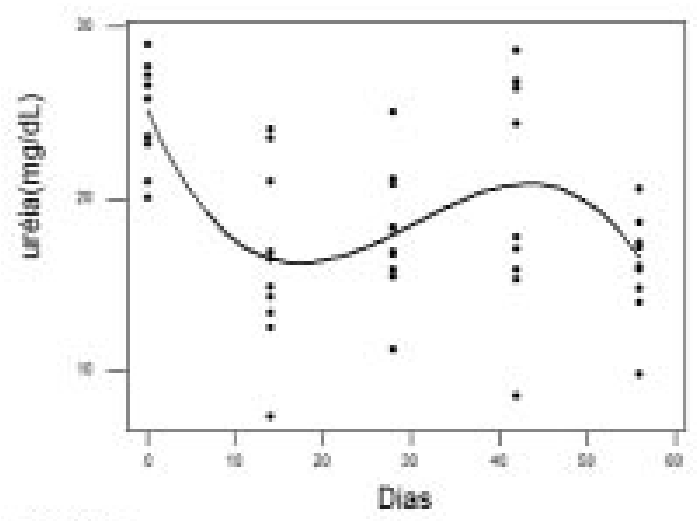

$\mathrm{R}^{2}=36,146$

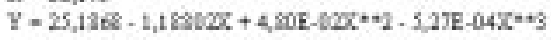

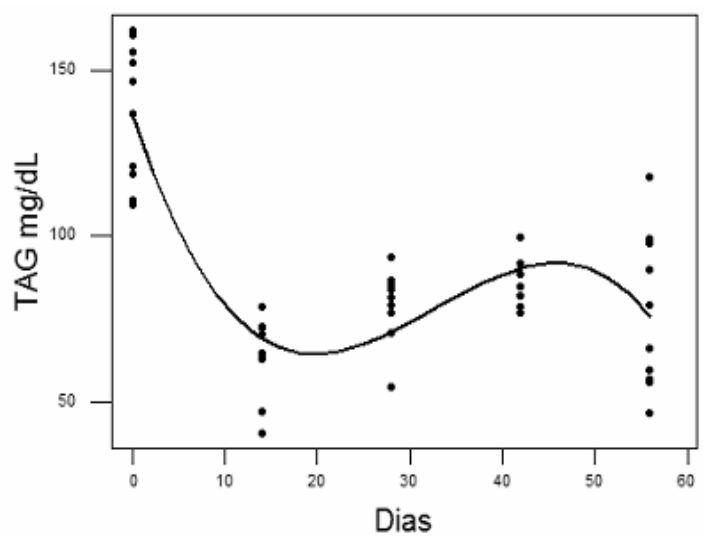

$\mathrm{R}^{2}=63,1 \%$

$\mathrm{Y}=132,107-5,28491 \mathrm{X}+0,182238 \mathrm{X} * *_{2}-1,92 \mathrm{E}-03 \mathrm{X} * * 3$

Figura 1: Análises de regressão das concentrações de ureia e TAG de touros semiconfinados submetidos a tratamento com rbST, ajustadas em função do período de coleta. 
Placebo

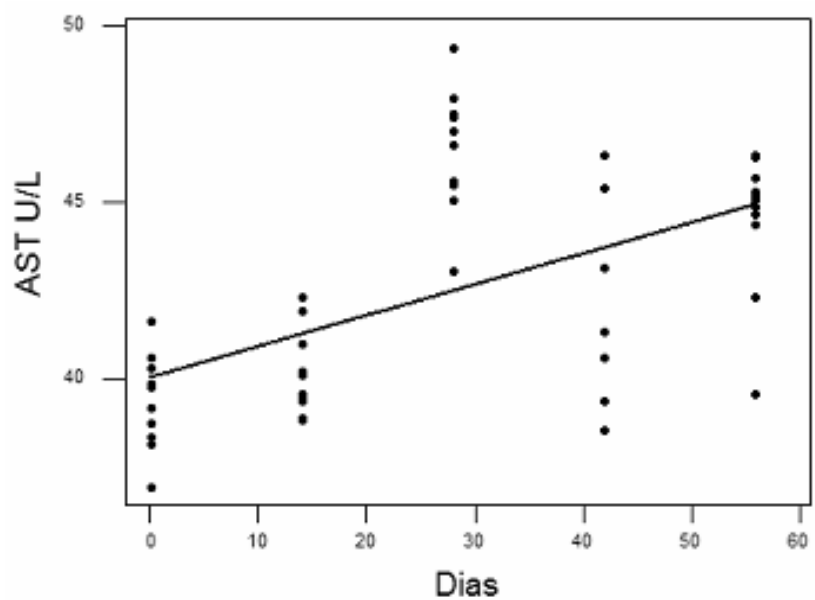

$\mathrm{R}^{2}=29,3 \%$

$Y=40,0866+8,73 \mathrm{E}-02 \mathrm{X}$

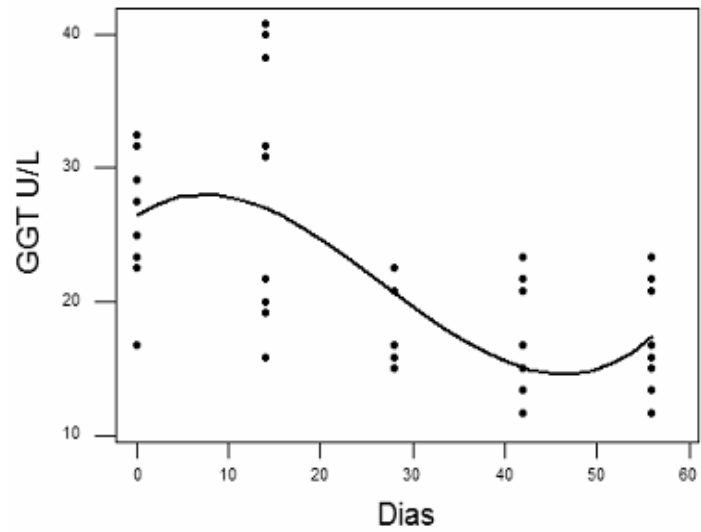

$\mathrm{R}^{2}=41,1 \%$

$\mathrm{Y}=26,4700+0,452000 \mathrm{X}-3,61 \mathrm{E}-02 \mathrm{X} * * 2+4,49 \mathrm{E}-04 \mathrm{X} * * 3$
rbST

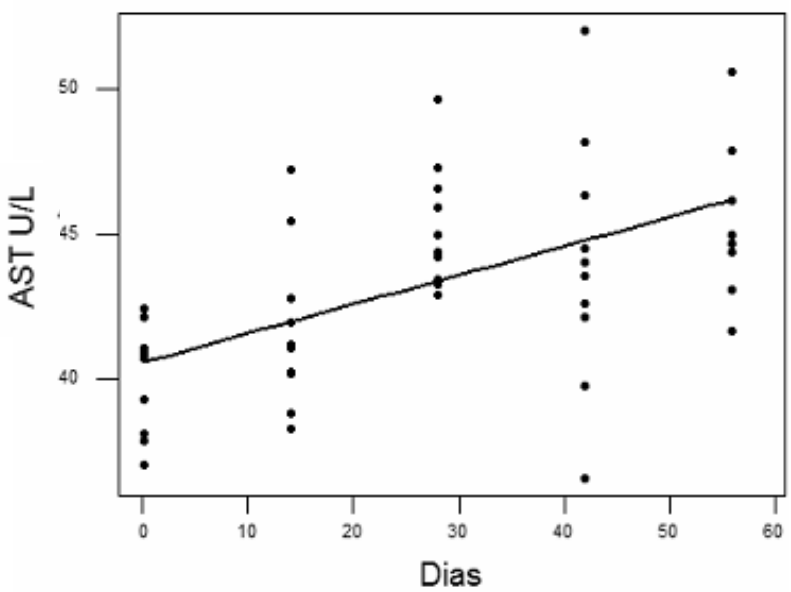

$\mathrm{R}^{2}=31,8 \%$

$Y=40,5672+0,100402 X$

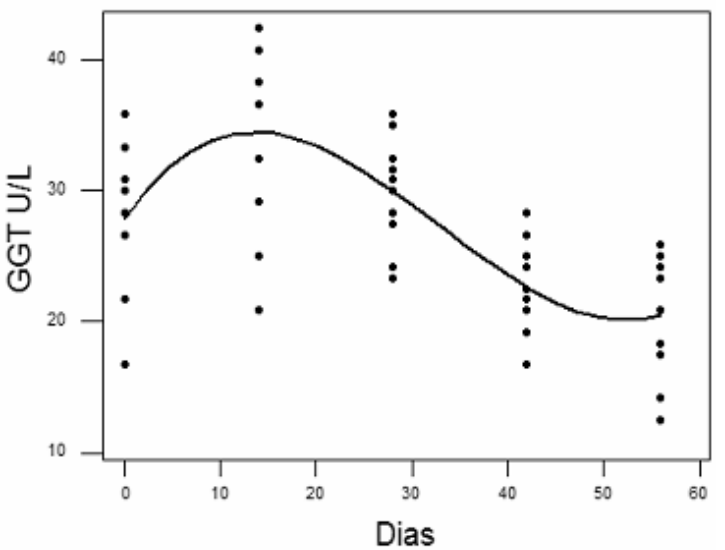

$\mathrm{R}^{2}=50,5 \%$

$\mathrm{Y}=27.8452+1.06179 \mathrm{X}-4.91 \mathrm{E}-02 \mathrm{X} * * 2+4.96 \mathrm{E}-04 \mathrm{X} * * 3$

Figura 2: Análises de regressão das concentrações de AST e GGT de touros semiconfinados submetidos a tratamento com rbST, ajustadas em função do período de coleta.

Através das análises de regressão (Figuras 1 e 2), pode-se observar que os marcadores bioquímicos avaliados apresentaram um comportamento sensível durante as coletas, com variações determinadas, por exemplo, pelo efeito cúbico $(P<0,05)$ demonstrado pelos marcadores TAG e GGT em ambos os grupos. Estas oscilações também foram observadas nas concentrações de ureia, que apresentou efeito quadrático no $\mathrm{Gl}$ e cúbico no Gll $(\mathrm{P}<0,05)$. O uso de rbST provavelmente promoveu um melhor aproveitamento dos substratos para gliconeogênese, pelo efeito que este hormônio tem sobre estas rotas (Pámela e Harvey, 1997), o que pode ser explicado pela diminuição nos níveis de TAG e ureia e aumento concomitante dos níveis de GGT no início do experimento. Possivelmente houve uma adaptação às condições de coleta de sangue, o que pode ter gerado uma condição de estresse e consequente aumento dos níveis de GH endógeno nos dois grupos (McCowen et al., 2001), o que também pode explicar a queda nos níveis de TAG e ureia entre os dias 0 e 30 do experimento, com posterior retomada de seus níveis. $O$ único marcador apresentando efeito linear
$(P<0,05)$ em ambos os grupos foi a AST, demonstrando ser um marcador mais estável da função hepática.

De acordo com os resultados, a utilização do rbST não provocou alterações significativas nos marcadores avaliados, demonstrando que não houve prejuízo ao equilíbrio metabólico destes touros, o que é importante para a atividade produtiva e reprodutiva dos animais. Assim, este tratamento hormonal pode ser realizado em touros sem causar transtornos metabólicos.

\section{Conclusão}

Os resultados obtidos demonstram que o tratamento com rbST não modificou os marcadores energéticos, proteico e minerais, apenas influenciando nas concentrações da enzima hepática GGT, o que não ocorreu com a AST. Estes resultados indicam que a utilização do rbST em touros não alterou a homeostasia, pois os parâmetros avaliados mantiveram-se de acordo com os padrões fisiológicos. 


\section{Referências}

BREIER, B.H. Regulation of protein and energy metabolism by the somatotropic axis. Domestic Animal Endocrinology, v. 17, p. 209-218, 1999.

BUTLER, W.R. Nutritional interactions with reproductive performance in dairy cattle. Animal Reproduction Science, v. 60, p. 449-457, 2000.

EISEMAN, J.H. et al. Effect of bovine growth hormone administration on metabolism of growing Hereford heifers: protein and lipid metabolism and plasma concentrations of metabolites and hormones. Journal of Nutrition, v. 116, p. 2504-2515, 1986.

GONG, J.G. Influence of metabolic hormones and nutrition on ovarian follicle development in cattle: practical implications. Domestic Animal Endocrinology, v. 23, p. 229-241, 2002.

GONZÁLEZ, F.H.D. et al. Uso do perfil metabólico para determinar o status nutricional em gado de corte. In: Perfil metabólico em ruminantes: Seu uso em nutrição e doenças nutricionais. Porto Alegre: UFRGS, 2000, p. 280-300.

GONZÁLEZ, F.H.D.; SILVA, S.C. Introdução à bioquímica clínica veterinária. Porto Alegre: UFRGS, 2003, p. 67-79.

GRUMMER, R.R.; CARROLL, D.J. Effects of dietary fat on metabolic disorders and reproductive performance of dairy cattle. Journal of Animal Science, v. 69, p. 3838-3852, 1991.

HOLZER, Z. et al. The effects of long-term administration of recombinant bovine somatotropin (posilac) and synovex on performance, plasma hormone and amino acid concentration, and muscle and subcutaneous fat fatty acid composition in holstein-friesian bull calves. Journal of Animal Science, v. 77, p. 1422-1430, 1999.

HULL, K.L.; S. HARVEY. Growth hormone: roles in male reproduction. Endocrine, v. 13, p. 243-250, 2000.

KAISER, G.G. et al. Effects of growth hormone on female reproductive organs. Anatomia, Histologia, Embryologia, v. 30, p. 265-271, 2001.

KANEKO, J.J. Clinical Biochemistry of Domestic Animals. 5th ed. San Diego: Acad. Press, 1997. p. 932.

KOLB, E. Fisiologia veterinária. 4. ed. Rio de Janeiro: Guanabara Koogan, 1984, p. 625.

LAPIERRE, $H$. et al. Effect of human growth hormone-releasing factor and (or) thyrotropin-releasing factor on growth, carcass composition, diet digestibility, nutrient balance, and plasma constituents in dairy calves. Journal of Animal Science, v. 69, p. 587-598, 1991.

LEHNINGER, A.L. et al. Princípios de Bioquímica. Editora Sarvier, 1995. p. 126-140.

LOWMAN, B.G. et al. Condition scoring beef cattle. Edingburgh: East of Scotland College of Agriculture (Bulletin 6), 1976. p. 8-39.
McCOWEN, K.C. et al. Stress-induced hyperglycemia. Critical Care Clinics, v.17, p. 107-124, 2001.

MARCHAL, R. et al. Effect of growth hormone (GH) on in vitro nuclear and cytoplasmic oocyte maturation, cumulus expansion, hyaluronan synthases, and connexins 32 and 43 expression, and $\mathrm{GH}$ receptor messenger RNA expression in equine and porcine species. Biology of Reproduction, v. 69, p. 1013-1022, 2003.

NATIONAL RESEARCHCOUNCIL-NRC. Nutrient requirements of beef cattle. 7. ed. Washington, D.C.: National Academy Press, 1998, p. 381.

ORR, C.C. Serum cooper, zinc, calcium and phosphorus concentrations of calves stressed by bovine respiratory disease and infectious bovine rhinotracheitis. Journal of Animal Science, v. 68, p. 2893- 2900, 1990.

PAMBU-GOLLAH, R. et al. An evolution for the use of blood metabolite concentrations as indicator of nutritional status in free-ranging indigenous goats. South Afric. Journal of Animal Science, v. 30, p. 115-120, 2000.

PAMELA, C.C.; HARVEY, R.A. Metabolismo dos ácidos graxos e trigliceróis. In: Bioquímica llustrada. 2. ed. Porto Alegre: Artes Médicas, 1997, p. 177-196.

PAYNE, J.M.; PAYNE, S. The metabolic profile test. Oxford: Oxford University, 1987. p. 123-147.

PRADO, I.N. et al. Somatotropina Bovina Recombinante (rbST) nos Aspectos Hematológicos e Metabólitos do Sangue de Novilhas ( $1 / 2$ Nelore x $1 / 2$ Red Angus) em Confinamento. Revista Brasileira de Zootecnia, v. 32, p. 465-472, 2003.

SAUERWEIN, H. et al. Growth hormone treatment of breeding bulls used for artificial insemination improves fertilization rates. Domestic Animal Endocrinology, v. 18, p. 145-158, 2000.

SCOTT, D. et al. Effect of diets varying in nitrogen or phosphorus content on indicators of bone growth in lambs. Experimental Physiology, v. 82, p. 193-202, 1997.

SIROTKIN, A.V. Control of reproductive processes by growth hormone: extra- and intracellular mechanisms. Veterinary Journal, v. 170, p. 307-317, 2005.

STORER, W.A. et al. The effects of equine somatotropin on pituitary and testicular function in the stallion during the nonbreeding season. Journal of Equine Veterinary Science, v. 25, p. 106-112, 2005.

UNDERWOOD, E.J. The mineral nutrition of livestock. 2. ed. Farnham Royal: CAB, 1981, p.102-103.

VASQUEZ, E.F.A.; HERRERA, A.P.N. Concentração plasmática de cortisol, ureia, cálcio e fósforo de vacas mantidas a pasto suplementadas com levedura de cromo durante a estação de monta. Ciência Rural, v. 33, p. 743-747, 2003.

WITTNER, F. et al. Determinación de úrea muestras de leche de rebaños bovinos para el diagnóstico de desbalance nutricional. Archivos de Medicina Veterinaria, v. 25, p. 165-172, 1993. 